\title{
AVALIAÇÃO DE DIFERENTES MÉTODOS DE ESTIMATIVA DE PARÂMETROS CINÉTICOS NA DEGRADAÇÃO TÉRMICA DE ANTOCIANINAS PRESENTES NA POLPA DE MORANGO
}

\author{
N. L. SILVA ${ }^{1}$, J. M. S. CRISPIM ${ }^{1}$ e R. P. VIEIRA ${ }^{1}$ \\ ${ }^{1}$ Instituto Federal de Educação Ciência e Tecnologia do Sul de Minas Gerais, Campus Pouso \\ Alegre \\ E-mail para contato: ronierik.vieira@ifsuldeminas.edu.br
}

\begin{abstract}
RESUMO - Este trabalho apresenta uma avaliação de três diferentes métodos para estimativa de parâmetros cinéticos da degradação térmica de antocianinas presentes na polpa de morango. O método integral, utilizando um modelo de primeira ordem se mostrou o mais adequado para representar este processo. Os resultados obtidos para a degradação de antocianinas assemelham-se a outros trabalhos disponíveis na literatura, confirmando que o modelo cinético proposto é adequado à esta situação.
\end{abstract}

\section{INTRODUÇÃO}

As antocianinas (de origem grega anthos, flor e kianos, azul escuro) são responsáveis pela coloração observada em frutas, flores algumas folhas sendo os pigmentos mais importantes após a clorofila (Harborne \& Grayer, 1988). Possuem elevada importância na dieta humana, auxiliando na prevenção de doenças cardiovasculares, certos tipos de câncer, somando-se à isto seu poder antioxidante é responsável no combate contra os radicais livres, sendo utilizadas também para fins terapêuticos (Ross e Kasum, 2002).

O morango, um pseudofruto que apresenta antocianinas, normalmente é industrializado na forma de geléias, pois apresenta perda na sua qualidade após a colheita, assim seu fornecimento ao mercado in natura torna-se limitado. Estes produtos processados também apresentam vida de prateleira relativamente curta, com elevadas percas na cor e no sabor, proporcionais ao tempo e à temperatura na qual são submetidos (Vendruscolo e Vendruscolo 2007).

A obtenção de um modelo cinético capaz de representar a degradação das antocianinas poderá auxiliar em pesquisas futuras afim de prever esta redução e assim aumentar o tempo de vida de prateleira e qualidade do produto industrializado. Esta reação pode ser representada simbolicamente por:

$$
\mathrm{A} \rightarrow \text { produtos }
$$

Portanto objetivo deste trabalho consiste na avaliação de diferentes métodos de estimativa de parâmetros cinéticos na degradação térmica de antocianinas presente em 
polpas de morango para a obtenção de um modelo adequado à reprodução dos dados experimentais. As análises aqui realizadas podem ser utilizadas em outros trabalhos afim de aprimorar processos de degradação de antocianinas que ocorrem a nível industrial.

\section{MATERIAIS E MÉTODOS}

Os dados experimentais de concentração de antocianinas em função do tempo para diferentes temperaturas $\left(60,70,80\right.$ e $\left.90^{\circ} \mathrm{C}\right)$ foram obtidos por meio do método do $\mathrm{pH}$ diferencial (Lee et al., 2005) utilizando a técnica de UV-Vis. Dispondo-se dos dados experimentais, três métodos de análise de dados cinéticos foram utilizados para ajustar o modelo e estimar seus parâmetros: o método diferencial, regressão não-linear e, finalmente, o método integral.

\subsection{Método diferencial}

Neste método, uma linearização direta da equação de velocidade genérica proposta como modelo (Equação 1) foi feita de modo avaliar todos os parâmetros da equação .

$$
\frac{-d C_{A}}{d t}=k C_{A}^{n}
$$

Em que $\mathrm{C}_{\mathrm{A}}$ representa a concentração de antocianinas $\left(\mathrm{mg} \mathrm{L}^{-1}\right)$ no tempo $\mathrm{t}, \mathrm{k}$ é a constante cinética de degradação do composto que depende da temperatura de acordo com a equação de Arrhenius (Equação 5), n é a ordem da reação de degradação. Logaritmando ambos os lados da Equação 1 e após manipulações matemáticas chega-se à Equação 2.

$$
\ln \left(\frac{-d C_{A}}{d t}\right)=\ln k+n \ln C_{A}
$$

Plotaram-se diferentes gráficos do logarítmo da taxa média de reação em função da concentração média para cada temperatura $\left(60,70,80\right.$ e $\left.90^{\circ} \mathrm{C}\right)$, através da substituição dos dados experimentais na Equação 2. O coeficiente linear da equação de reta obtida forneceu o valor da constante cinética de degradação do composto, $\mathrm{k}$, e o coeficiente angular representou a ordem da reação, n. A eficácia do método foi determinada através do coeficiente de correlação linear $\left(\mathrm{R}^{2}\right)$, cujo valor desejável foi acima de 0,9 .

\subsection{Regressão não - linear}

O ajuste de curvas utilizando regressão não-linear foi realizado utilizando o método dos mínimos quadrados, com o auxílio do SOLVER do Excel, a fim de minimizar a Equação 3, para todos os dados experimentais disponíveis.

$$
\sum_{1}^{i}\left(t_{\exp }-t_{c a l}\right)^{2}
$$


Em que $t_{\text {exp }}$ é tempo obtido experimentalmente, $t_{\text {cal }}$ corresponde ao tempo calculado pela Equação 4, que é a forma integrada da Equação 1, i representa o número de dados experimentais disponíveis.

$$
t_{\text {calc }}=\frac{C_{A \exp }^{1-n}-C_{A 0}^{1-n}}{(n-1) k}
$$

Em que $\mathrm{C}_{\text {Aexp }}$ representa a concentração de antocianinas $\left(\mathrm{mg} \mathrm{L}^{-1}\right)$ obtida experimentalmente no tempo $\mathrm{t}, \mathrm{C}_{\mathrm{A} 0}$ a concentração inicial de antocianinas, $\mathrm{n}$ a ordem da reação, $\mathrm{k}$ a constante cinética de degradação do composto. $\mathrm{O}$ valor mínimo da soma dos quadrados dos resíduos (Equação 3), variando os parâmetros k e n, através do método GRG, do Solver forneceu a ordem da reação e a constante cinética mais adequadas para cada temperatura.

\subsection{Método Integral}

O método consistiu na linearização dos dados experimentais e ajuste às equações de taxa integradas e linearizadas de três modelos cinéticos distintos: o modelo de ordem zero, um e dois. Uma avaliação visual de cada modelo, juntamente com a análise do coeficiente de determinação $\left(\mathrm{R}^{2}\right)$ forneceram direcionamentos para a escolha do modelo mais adequado.

\subsection{Determinação do fator pré - exponencial e energia de ativação}

De acordo com a equação de Arrhenius (Equação 5) a constante de taxa, k, depende da temperatura .

$$
k=k_{0} e^{-E a / R T}
$$

Em que $\mathrm{k}_{0}$ é o fator pré-exponencial $\left(\mathrm{min}^{-1}\right), \mathrm{E}_{\mathrm{a}}$ é a energia de ativação $\left(\mathrm{J}^{\mathrm{mol}}{ }^{-1}\right)$ e $\mathrm{R}$ a constante dos gases $\left(8,314 \mathrm{~J} \mathrm{~mol}^{-1} \mathrm{~K}^{-1}\right) . \mathrm{k}_{0}$ e $\mathrm{E}_{\mathrm{a}}$ foram estimados através da linearização da Equação 5 e ajuste para as temperaturas $60,70,80$ e $90^{\circ} \mathrm{C}$.

\section{RESULTADOS E DISCUSSÃO}

A estimativa através do método diferencial não forneceu resultados satisfatórios, pois as equações de reta forneceram coeficientes de correlação linear $\left(\mathrm{R}^{2}\right)$ todos abaixo de 0,9. Este resultado pode estar associado ao fato do método em questão fornecer uma aproximação para a derivada da equação de taxa, ou seja, valores médios. Estes valores médios podem ser bastante imprecisos em virtude dos pontos experimentais terem sido coletados em intervalos de tempo não muito próximos. 
O método de regressão-linear apresentou valores de ordem de reação $n$ entre 1,03 - 1,31, próximo ao método integral, que por sua vez apresentou um bom ajuste no modelo de primeira ordem. Entretanto, os valores das constantes de reação $k$ se diferenciaram bastante nos dois métodos considerados. É importante ressaltar que o ajuste não linear possivelmente necessitaria de boas atribuições iniciais para as constantes para que o método não convergisse em um mínimo local da função objetivo. Em virtude deste fato e, também, da simplicidade e precisão encontradas ao método integral, este será utilizado para estimar todos os parâmetros do modelo cinético.

O melhor ajuste foi no modelo de primeira ordem, como já citado anteriormente. Assim, considerando um reator em batelada como sistema, integrou-se a Equação 6, obtendo-se a Equação 7.

$$
\begin{aligned}
& \frac{d C_{A}}{d t}=-k C_{A} \\
& C_{A}=C_{A 0} e^{-k t}
\end{aligned}
$$

Em que $\mathrm{C}_{\mathrm{A}}$ representa a concentração de antocianinas $\left(\mathrm{mg} \mathrm{L}^{-1}\right)$ no tempo t, $\mathrm{C}_{\mathrm{A} 0}$ é a concentração inicial de antocianinas e k é a constante cinética de degradação .

A Figura 1 representa a relação entre $\ln \left(\mathrm{C}_{\mathrm{A}} / \mathrm{C}_{\mathrm{A} 0}\right)$ em função do tempo em minutos. Observa-se que os coeficientes de correlação linear $\left(\mathrm{R}^{2}\right)$ foram aproximadamente 0,99, logo, comprova-se que o modelo cinético de primeira ordem representa satisfatoriamente a degradação térmica de antocianinas presentes na polpa de morango. Resultados semelhantes foram encontrados nos trabalhos de Miguel et al. (2009), na análise da degradação da cor em geléia de morango, e por Skrede et al. (1992) que analisou a velocidade de degradação de antocianinas em xarope de morango.

Figura 1 - Logarítmo natural da relação $\mathrm{C}_{\mathrm{A} 0} / \mathrm{C}_{\mathrm{A}}$ em função do tempo de reação

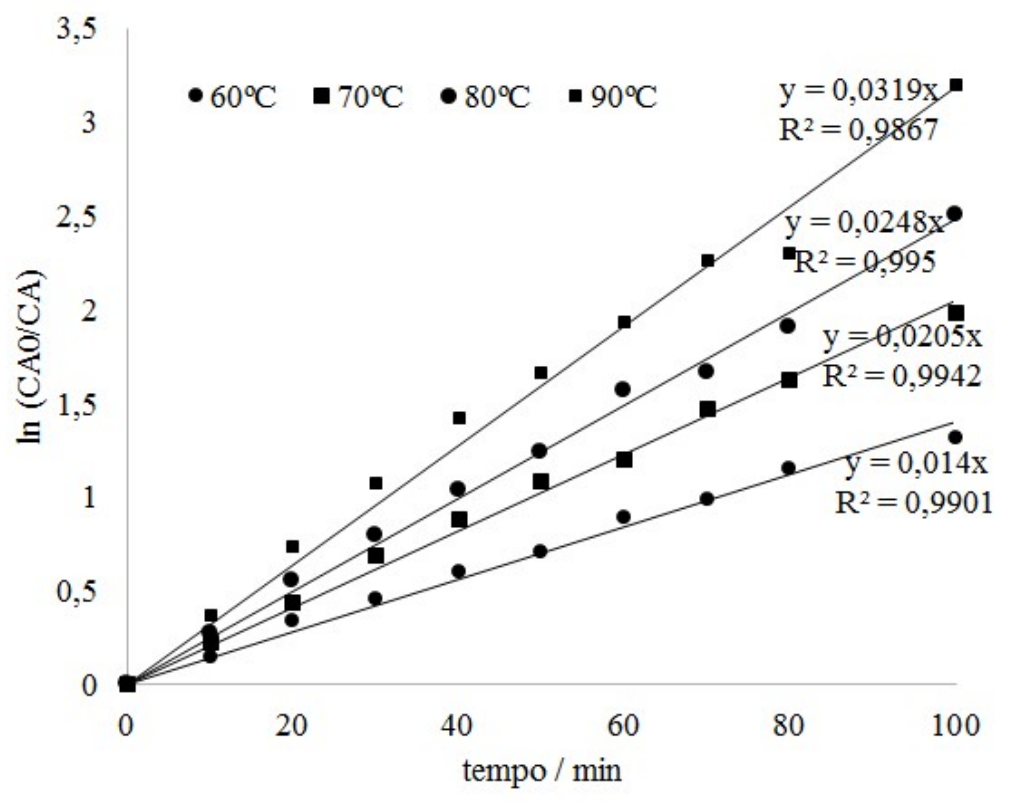


Analisando-se a Figura 1, verifica-se que em uma mesma temperatura as concentrações de antocianinas foram diminuindo com o passar do tempo de exposição ao aquecimento, e para o mesmo tempo em temperaturas distintas, as concentrações diminuiram cada vez mais em temperaturas maiores.

Através das equações características das retas na Figura 1 obtem-se as constantes de taxa para diferentes temperaturas $\left(60,70,80\right.$ e $\left.90^{\circ} \mathrm{C}\right)$. A partir destas podemos determinar os parâmetros da equação de Arrhenius (energia de ativação e a fator pré-exponencial) conforme a Figura 2 . Os valores dos parâmetros encontrados foram $\mathrm{k}_{0}=233,71 \mathrm{~min}^{-1} \mathrm{e} \mathrm{E}_{\mathrm{a}}=26829,28 \mathrm{~J}$. $\mathrm{mol}^{-1}$ utilizando-se a Equação 5 em sua forma linearizada. Comparando-se a energia de ativação de 5,66 kcal.mol ${ }^{-1}\left(23681,44 \mathrm{~J} \mathrm{~mol}^{-1}\right)$ encontrada no trabalho de Miguel et al. (2009), ao se avaliar a degradação da cor em geleiada de morango, verificou-se que os resultados do presente trabalho seguem esta mesma tendência.

Figura 2 - Dependência das constantes cinéticas em relação à temperatura.

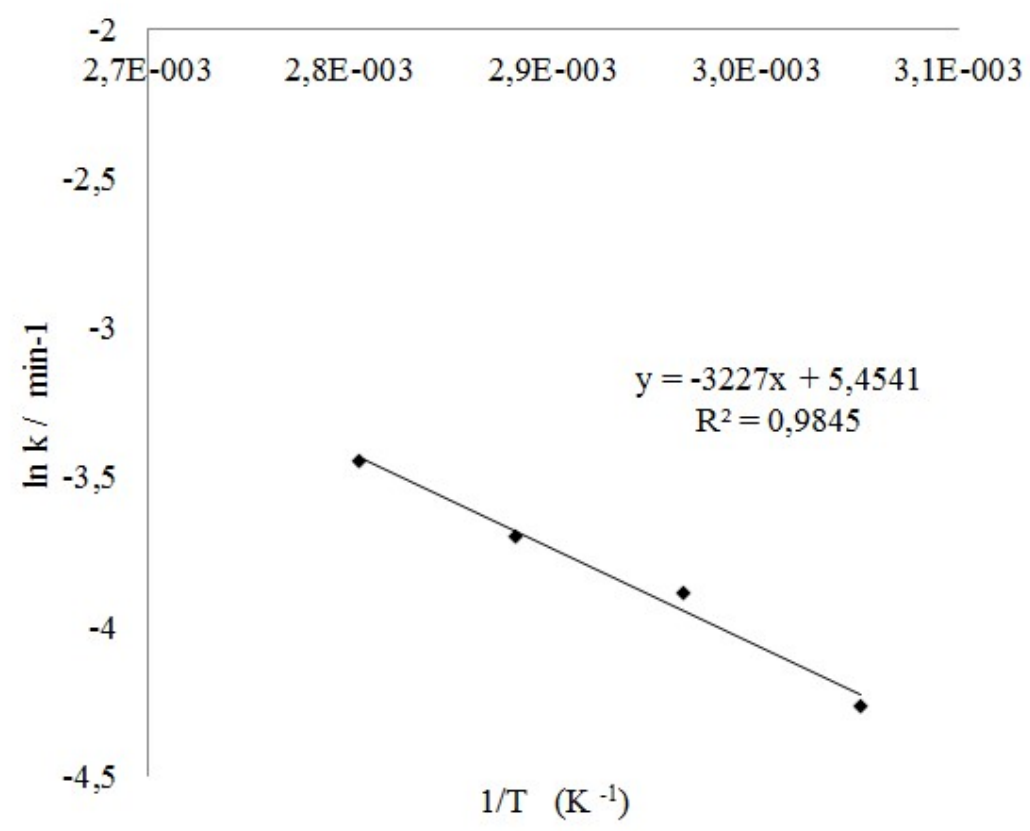

\section{CONCLUSÃO}

O objetivo deste trabalho consistiu em fornecer um modelo que melhor representasse a degradação térmica de antocianinas presentes na polpa de morango. O modelo integral de primeira ordem melhor de adequou aos dados experimentais. Utilizando-se a Equação de Arrhenius obtemos os seguintes parâmetros $\mathrm{k}_{0}$ igual a 233,71 $\min ^{-1} \mathrm{e}$ a energia de ativação $\mathrm{E}_{\mathrm{a}}$ 26829,28 J. mol ${ }^{-1}$, próximos aos valores descritos na literatura em outros trabalhos, validando nosso modelo. Os resultados aqui obtidos podem ser utilizados na otimização de processos a nível industrial.

\section{AGRADECIMENTOS}

Os autores agradecem ao Instituto Federal do Sul de Minas Gerais, Campus Pouso 
Alegre - IFSULDEMINAS, pelo transporte fornecido à participação no evento.

N. L. da Silva agradece à Fundação de Amparo à Pesquisa de Minas Gerais (FAPEMIG) pela bolsa de IC concedida.

\section{REFERÊNCIAS}

HARBORNE, J.B.; GRAYER, R.J., The anthocyanins. In: The flavonoids: advances in research since 1980. Chapmam \& Hall, London, 1988, p. 1-20.

LEE, J., DURST, R.W. , WROLSTAD, R.E., 2005. Determination of Total Monomeric Anthocyanin Pigment Content of Fruit Juices, Beverages, Natural Colorants, and Wines by the pH Differential Method: Collaborative Study. J. AOAC Int. 88, 1269-1278.

MIGUEL A.C.A.; ALBERTINI S.; SPOTO M.H.F.; Cinética da degradação de geleiada de morango. Ciência e Tecnologia de Alimentos 2009, 29(1): 149-7.

ROSS, J.A.; KASUM, C.M. Dietary flavonoids: bioavailability, metabolic effects, and safety.Annual Review of Nutrition, Palo Alto, n.22, p.19-34, 2002.

SKREDE, G. et al. Color stability of strawberry and blackcurrant syrups. Journal of Food Science, v. 57, n. 1, p. 172-177, 1992.

VENDRUSCOLO, J. L. S.; VENDRUSCOLO, C. T. Sistema de produção do morango.Disponívelem: $<$ http://sistemasdeproducao.cnptia.embrapa.br/FontesHTML/Morang o/SistemaProducaoMorango/cap14.htm>. Acesso em: 18 Março 2017. 